\title{
Workers' task choice heuristics as a source of emergent structure in digital microwork
}

\author{
Jean-Michel Dalle \\ Sorbonne Université, i3- \\ CRG (Ecole Polytechnique \\ and $C N R S)$, \\ Oxford Internet Institute
}

Otto Kässi

Vili Lehdonvirta

Oxford Internet Institute

Oxford Internet Institute,

Alan Turing Institute

Digital labor markets are structured around tasks and not around fixed-or long-term employment contracts. We study the consequences of the granularization of work for digital micro workers. To address this question, we combine interview data from active online micro workers and online data on open projects scraped from Amazon's Mechanical Turk platform to study how the digital micro workers choose which tasks they work on. We find evidence for preferential attachment: workers prefer to attach themselves to experienced employers who are known to offer high quality projects. In addition, workers also clearly prefer long series of repeatable tasks over one-off tasks, even when one-off tasks pay considerably more. We thus see a re-emergence of certain types of organizational structure.

Keywords: Microwork, online platforms, problem of problem choice, task choice

JEL: J22, J41, J46 


\section{Introduction}

A structural difference between online labor markets and their offline and more traditional counterparts comes from the fact that many of the new online labor markets are structured around tasks and not around fixed- or long-term employment contracts. Indeed, the new digital platforms fundamentally "shape the terms on which participants interact with one another" (Kenney \& Zysman, 2016); online workers do not look for a job but choose sequentially among available tasks. A prominent example of this is Amazon Mechanical Turk ("MTurk"), the oldest and one of the most prominent microwork markets.

A consequence of the evolution towards predominantly task-based labor markets is that it alters the structure of switching costs that workers face when they change from one task to another, again compared to changing from one job to another in a more traditional employment context. The micro-strategies according to which agents decide to take a new job, or to wait for other, potentially better opportunities have long been a central topic of interest in economics and social sciences. Indeed, the intricacies of "job search" have been found to bear severe consequences at the aggregate level, notably with respect to otherwise unexplainable phenomena related to unemployment dynamics and wage stickiness (Diamond 1982, Pissarides 1985).

What, now, when labor markets are becoming digital and task-based? What consequences does the granularization of tasks have on the aggregate dynamics? We argue that this evolution, from job search to task choice, warrants specific investigations with respect to the micro-behaviors of online workers and on their aggregate consequences in online labor markets. Different switching cost structures and the increased granularity of online work certainly affect the collective allocation of efforts by workers - the supply of online labor at the aggregate level in ways that are not straightforward or easy to guess without direct empirical studies.

Which tasks do online workers select? How do the heterogeneous motivations, incentives, reward structures, etc., of online workers match with the characteristics of the tasks offered, and how does it orient their choices, individually and collectively? We believe that this issue needs to be approached using a mixed-methods, qualitative and quantitative, empirical approach. This is what we try to provide in this article. We first put the issue of task choice in online markets in perspective (Section 2). We then report on a qualitative study about the behaviors of online workers based on field interviews (Section 3). Section 4 discusses the details of the collection of quantitative data. In Section 5, we turn to regression analyses, which highlight the strong preference towards so-called "batch" tasks - large numbers of similar, 
simple tasks - in the choices of workers, in line with the results of the field study and with the findings of other recent studies. This salience of batch tasks is strong enough that the pricing of individual tasks is only weakly associated with labor supply. We conclude by discussing these findings with regard to their aggregate consequences to both academics using MTurk and to online labor markets in general.

\section{The problem of task choice}

Jobs can be seen as bundles of tasks. In the standard 20th-century model of employment, tasks were generally bundled into chunks that correspond in size with full-time employment. All the tasks were in service of a single employer, the work being typically performed during regular working hours, and associated with an expectation of continued employment (Ashford et al. 2007). Furthermore, in this standard employment model, the composition of a worker's task bundle is mostly not up to the worker to choose but is instead determined by exogenous factors such as industry, work organization, technology, and management (Acemoglu \& Autor 2011, Autor \& Handel 2013). Workers choose which role and thus task bundle to commit to, after which their choice is limited.

However, this standard model of employment has become increasingly less prevalent over the past few decades (Kalleberg 2000, Kalleberg 2011). Job tenures have become shorter and changing from one occupation to another has become more common (Hollister 2011). Different forms of non-standard employment have emerged and grown alongside standard employment, including forms that are discussed under such terms as contingent work (Connelly \& Gallagher 2004), freelancing (Gold \& Mustafa 2013), professional contracting (Barley \& Kunda 2006), and portfolio work (Fraser \& Gold 2001). These non-standard forms of employment are characterized by the unbundling of jobs into gigs, projects, shifts, and tasks of increasing granularity. This unbundling or disaggregation is at least partly driven by the adoption of new technologies that make it economical to engage workers on increasingly flexible, just-in-time, on-demand, and market-based contracts (Malone 2004, Kalleberg 2011, Sundararajan 2016).

An extreme example of the unbundling of jobs into tasks is found on the online piecework platform Amazon Mechanical Turk (Bergvall-Kåreborn \& Howcroft 2014, Lehdonvirta 2018). MTurk is a website initially launched in 2005 by e-commerce giant Amazon as a way to source remote contingent workers for the company's needs, and subsequently opened for outside employers. Employers post self-contained "microtasks" such as transcribing a sales receipt or adding content descriptors to a video clip. Workers browse the tasks and pick tasks to complete, earning from a few cents to a few dollars per task. There are thousands of tasks to choose from 
at any time, and each task typically takes from seconds to up to hours to complete. In contrast with the standard employment model, workers in MTurk retain full choice over the composition of their task bundles. They weave the exact sequence of their work by repeatedly choosing the next task to work on, from the full universe of tasks, on a task-by-task basis. They also choose when to start completing tasks and for how long. Instead of a hierarchical employment relationship, MTurk thus exemplifies a spot market for tasks (Barley et al. 2017, Oyer 2016).

To understand why this unbundling of tasks, and the generalization of task choice as its consequence, can have important aggregate consequences, consider the analogous issue in science, known in the sociology of science as the "problem of problem choice" (Merton, 1957; Zuckerman, 1978; Ziman, 1987). Among the most important decisions an academic has to make is the choice over which scientific problem they will investigate. Are they going to try to address a new or difficult problem, risky but with a potentially great reward in case they manage to contribute to significant progress? Or will they prefer an active problem that almost guarantees easy publication of incremental results, but with only limited rewards in terms of reputation? This simple but prevalent issue goes back to Peirce (1896), who first saw that there was an important point to be addressed with respect to the allocation of efforts between different projects based on their characteristics ${ }^{1}$.

More generally, as soon as there exists some degree of freedom granted to agents when deciding on their agendas, that is, on which tasks they will allocate their efforts, agents will self-select in relation to the menu of tasks and to their often partly ignored individual characteristics, and also in relation to choices by others who influence at the very least the rewards of existing available tasks and who further often convey relevant if not meaningful information. Contributors to various online communities, such as Wikipedia or open-source software, typically face similar issues, as they must choose to which project to contribute, and on which of the many tasks within their project to dedicate their efforts (Dalle \& David, 2005), and it has

${ }^{1}$ Carayol \& Dalle (2007) have modelled this phenomenon at the level of a scientific community and taking into account the heterogeneity of academics, in order to evaluate the collective, aggregate consequences of the academic freedom in choosing individual research agendas - modulo motivations and incentives, as they result from the institutions of modern science - and with respect also to the potential social utility of the general body of knowledge thus produced. 
for instance been shown that developers in large projects tended to form teams mostly in relation to software code that includes the most complex software procedures, suggesting a preference of developers towards more difficult problems perhaps at the expense of more mundane and simpler, but perhaps very useful, tasks (Den Besten, Dalle \& Galia, 2008).

In the economic literature, the process by which workers match with jobs has been of particular interest for the "macro-job search" literature (Diamond 1982, Pissarides 1985). The starting point of the analysis in this literature is that, because workers care about various dimensions of their jobs, such as tasks, wages, and location, searching for good jobs takes considerable effort. Employers have similar concerns about their workers, and since jobs usually consist of large chunks of work, both parties are willing to expend considerable effort before committing to a match. As a consequence, virtually all national economies simultaneously exhibit vacancies and involuntary unemployment. The goal of search and matching literature has thus been to replicate and explain the macro-level dynamics of aggregate unemployment and vacancies, while abstracting away from the exact process of job matching.

We are aware of a handful of previous studies that directly or indirectly address the problem of task choice on granular online labor markets - Chilton et al. (2010) and Farenzana et al. (2016), the second explicitly replicating the results of the former, and Kaplan et al. (2018). In addition, a number of studies (e.g. Mason \& Watts 2010; Finnerty et al. 2013) have studied MTurk's viability as a research participant pool with a particular emphasis on how HIT rewards affect the rate of completion and quality of the results.

Chilton et al. (2010) study how the ways in which MTurk's user interface allows workers to sort tasks influences which tasks are completed fastest. They also survey workers for selfreported information about how they search for tasks. The results suggest that workers mostly sort by which tasks are most recently posted and by which tasks have the largest number of tasks available and focus on the first few pages of the resulting search results. Farenzana et al. (2016) further suggest that the process of search and evaluation of tasks plays an important role in how workers solve tasks of a short duration, also stressing that the way elements are arranged visually on search screens could play a role with respect to how workers choose. On their part, Kaplan et al. (2018) conducted a survey to study the strategies that online workers use to find and complete tasks, with a main focus on monetary earnings. Their observations suggest the main determinants of task choice are high pay and low effort. However, the authors note some elements of contradiction between these observations as their survey respondents also show a strong preference for working on batches of repetitive tasks, which usually are not well paid. 
In the context of using MTurk as a participant pool for social science research, Mason and Watts (2010) note that tasks with higher rewards tend to get completed faster, but might generate poorer quality results, while Finnerty et al. (2013) highlight that simpler tasks generate higher quality results independently of rewards.

The studies discussed above provide a number of interesting findings on task choice, but the findings are sometimes contradictory, and none of the studies is able to put forward a holistic picture or model of task choice behavior that would account for the differing results. They also do not address how different workers might self-select into different tasks, a phenomenon that could easily explain why both a preference for a higher pay and a preference for repetitive tasks was observed by Kaplan et al. (2018). In a heterogeneous population of workers, both preferences might coexist in ways that deserve to be understood if we are really to shed light on the aggregate consequences.

In order to move in this direction, we believe it is necessary to combine both qualitative and quantitative observations focused on MTurk, which is by far the most studied platform in the literature and a platform where the granularity of the tasks is particularly high, which makes it especially relevant with respect to the problem of task choice in a task-based online labor market.

\section{How do you turk? The heuristics of turking}

How do workers decide which tasks to pick out of the thousands available on MTurk? In this section, we address this question with qualitative evidence on MTurk workers' problem choice practices, based on interviews with ten MTurk workers. These interviews were supplemented with hands-on participant observation in the worker and employer roles, and with observations on workers' public online forums. The data were collected between 2012 and 2013 as part of a larger study (Lehdonvirta, 2016). We recruited the interview informants via a major workerrun online forum; the informants were thus likely to be more active users than typical. The informants were based in the United States ${ }^{2}$ and aged between 23 and 62. Eight were women. Informants quoted figures between 14 and 200 dollars as their typical weekly MTurk income.

\footnotetext{
${ }^{2}$ According to a the real-time demographics tracker of workers on MTurk (http://demographics.mturktracker.com/\#/countries/all accessed 2018-07-09), over 70\% of the workers are based in the United States.
} 
Three informants depended on MTurk and to a lesser extent on other online labor platforms for a significant part of their income. Others enjoyed a stable income from their own or their partner's standard employment and used MTurk to earn extra spending money. In these and other respects our interview informants thus represented a range of different demographic backgrounds and motivations, similar to American MTurk workers depicted in surveys (Hitlin et al., 2016). Interviews were conducted via teleconferencing (one informant) and via various text-based instant messaging channels (IM) used by the workers in their communications amongst each other (nine informants). A total of approximately 15 hours of interviews were conducted. Interview transcripts were coded with a two-stage iterative coding process to identify and categorize practices related to the problem of problem choice. The interviews were conducted without any prior hypotheses in mind related to problem choice, so the results reveal practices put forward by the informants on their own accord.

First of all, the interview findings support the notion that task choice is a very important problem for MTurk workers and that they use several different heuristics to find tasks. As one informant typically noted, "the work itself is not hard. Everything else surrounding [it] is what is hard to manage." The platform made available hundreds of thousands of tasks at any time, grouped into thousands of 'HIT groups'. The tasks were highly heterogeneous on multiple dimensions. The task content could be anything from transcribing business cards or answering questions in a marketing survey to writing an essay or recording speech or video. Complexity and skill requirements varied from simple cognitive tasks to tasks that required reasoning, creativity, or specialized knowledge or skills, such as language skills. Prices paid per task varied from one cent to several dollars, in rare cases exceeding $\$ 100$ per task. The time required to complete a task varied from seconds to hours. Workers' earnings were thus highly contingent on how well they managed to solve the problem of task choice.

In general, workers tried to choose among tasks in a way that maximized earnings over time. This meant choosing tasks that paid well relative to the time spent, and also minimizing unpaid search time. To some extent it also meant improving future earning potential by choosing tasks that promoted learning and skill development, but for the most part our informants operated on short time horizons, trying to maximize their daily or weekly earnings. A further constraint on search time was that workers on the platform were engaged in competition with each other for the best tasks: "we race to grab different types of HITs before other workers get them [...] you have to work fast. So fast things seem like a blur." Most informants were also simultaneously juggling other activities, such as other sources of work; three informants worked through their mobile phones, two of them during the working hours of their regular service sector jobs. 
Workers could evaluate tasks by reading task descriptions and looking at the prices posted. However, this was unlikely to produce accurate estimates of potential earnings. The amount of time required to complete a task could not be accurately deduced from its description; when the employer provided an estimate (common in survey tasks), it was often too low. Moreover, a task's earning potential also depended on the characteristics of the employer, such as whether and how quickly they responded to queries in case of problems, and how likely they were to reject work and decline to pay for it. For these and related reasons, the tasks were to a great extent risky choice: their value could be accurately determined only after the completion of the task.

Given that thousands of different types of tasks were available at any time, it was entirely unfeasible for a worker to perform a complete search, that is, to sample all the tasks to find the best types. As one informant put it, "There is a lot of poor work to wade through." To some extent workers shared information on good tasks through their social networks (see also Kingsley et al. 2015, Lehdonvirta 2018), but this was constrained by competition between the workers, and by the fact that workers' skills and thus their optimal tasks were to some extent idiosyncratic. New task types also constantly appeared and old ones disappeared. How, then, did workers choose which tasks to work on in this challenging environment? We observed the use of at least six heuristics, summarized in Table 1.

Table 1. Task choice heuristics among MTurk workers

\begin{tabular}{|l|l|l|}
\hline Heuristic & Example quotes & Freq \\
\hline Employer & $\begin{array}{l}\text { "I have a killer set of bookmarks to certain requesters." } \\
\text { "My requester base is made up of requesters that I know } \\
\text { pay fairly and are worth working for." }\end{array}$ & $\mathrm{n}=10$ \\
\hline Batch preference & $\begin{array}{l}\text { "They're batch HITs, so each only pays a few cents } \\
\text { maybe, but you hope to do a lot of them." }\end{array}$ & $\mathrm{n}=6$ \\
& $\begin{array}{l}\text { "One of my favorite feelings is hammering out a bunch } \\
\text { of those and then checking my total earnings for the day" }\end{array}$ & \\
\hline
\end{tabular}




\begin{tabular}{|c|c|c|}
\hline Specialization & $\begin{array}{l}\text { "I like to do sentiment analysis, you know, read this } \\
\text { tweet and say if it's positive or negative. I've been } \\
\text { getting more into content moderation and editing, too." } \\
\text { "I focus on them because I think they fit my particular } \\
\text { skillset, I feel good at them." }\end{array}$ & $\mathrm{n}=5$ \\
\hline Reservation wage & $\begin{array}{l}\text { "I do not work for less than } 10 \text { cents a minute." } \\
\text { "A lot of work pays way below minimum wage, and I } \\
\text { won't do that." }\end{array}$ & $\mathrm{n}=4$ \\
\hline $\begin{array}{l}\text { High-priced task } \\
\text { aversion }\end{array}$ & $\begin{array}{l}\text { "So many of the big dollar HITs are scams, I tend to } \\
\text { expect a big dollar figure means I should avoid it." }\end{array}$ & $\mathrm{n}=1$ \\
\hline $\begin{array}{l}\text { Short cycle } \\
\text { preference }\end{array}$ & "I only stick with the short HITs." & $\mathrm{n}=1$ \\
\hline $\begin{array}{l}\text { Limited task } \\
\text { search time }\end{array}$ & $\begin{array}{l}\text { "Must not spend more than fifteen minutes searching for } \\
\text { work, after that time if nothing is found I give up for at } \\
\text { least an hour or two." }\end{array}$ & $\mathrm{n}=1$ \\
\hline
\end{tabular}

All informants had preferred employers whose tasks they chose first. The informants had had previous good experiences with these employer's tasks, and expected subsequent tasks to be good, too. Though MTurk did not provide a formal reputation system for rating employers, some employers had thus in effect attracted a positive informal reputation among workers. Information on good employers was to some extent shared among workers; third-party website and browser plugin Turkopticon even provided a way for participating workers to formally rate employers on multiple dimensions and view ratings submitted by others (Irani \& Silberman 2013). However, relying on preferred employers only went so far as a decision heuristic. Preferred employers could only provide so many tasks, and there was significant employer turnover over time. "HITs come and go, requesters come and go. There is no way to make the same each day, weekly or monthly," observed one informant. 
After preferred employers, preference for so-called batch tasks stood out as the most prominent heuristic, in terms of the number of informants who spontaneously brought it up $(n=6)$ and the importance that they ascribed to it. One informant summed up the concept and its benefits thus:

"One of the types of HITs I like to work on are called batch HITs. They are generally low paying per HIT, but the HITs can be done quickly and are repetitive. Say it pays 8 cents per HIT, but only takes 10 seconds to do. That adds up quick. One of my favorite feelings is hammering out a bunch of those and then checking my total earnings for the day and seeing how much it has grown."

Batch tasks helped workers to maximize earnings over time by minimizing the unpaid search time. They also allowed the worker to hone their practices over successive iterations of the task, slightly improving their productivity over time. Two informants also alluded to psychological benefits similar to those discussed in Warr (1987), in that batch hits allowed the workers to "get into a rhythm" that made the work seem more effortless. A related heuristic was to prefer or specialize in certain types of task content, mentioned by five informants. Some workers specialized in writing tasks, some in sentiment analysis, and so on, regardless of the employer and of whether the tasks came in batches. This enabled some productivity gains from on-thejob learning.

A heuristic mentioned by four informants was to set a reservation wage, that is, to refuse tasks that resulted in earnings per minute or earnings hour below a certain threshold. This was mostly applicable surveys and similar tasks that indicated the expected duration in the description, allowing expected earnings to be estimated easily (if inaccurately). If the expected earnings exceeded the threshold, then the worker could stop the task search and accept the task. Informants also paid attention to tasks with large per-unit pay but expressed ambiguity over them. In the best case, a high-priced task could be a web content writing job taking an hour or so and paying a very good wage to a quick writer. In the worst case, a high-priced task could be not just a waste of time, but an attempt to trick the worker into revealing sensitive personal information for what were assumed to be nefarious purposes. One informant had developed a negative heuristic with regard to task prices: "I tend to expect a big dollar figure means I should avoid it."

One informant said that she preferred smaller tasks over bigger tasks, because they were easier to squeeze into her hectic schedule. In general, since many workers completed tasks in whatever slivers of unoccupied time they had, a short cycle time or the ability to drop the work at a moment's notice would have been important for them. This need for temporal flexibility might 
have also contributed to the popularity of batch tasks, which were always short in duration. Another informant had developed a rule where she would limit task search time to a maximum of 15 minutes per session.

Whenever workers found work that allowed them to earn well, they added the employer ('requester') in question to their list of preferred employers and started performing regular and in some cases automated checks to see if the requester had posted more work. Some had even learned the times of the day or days of the week that their preferred employers typically posted tasks. However, preferred employers often stopped posting tasks at some point, and in any case were only able to provide a part of the work necessary for sufficient earnings, especially for those who relied on MTurk for a significant part of their income. At that point, workers once again fell back on the other heuristics to choose tasks to work on.

Overall, these qualitative findings suggest that due to the institutional structure of the microtask market that breaks jobs into small tasks that are allocated on an individual basis, task search becomes a significant problem. Search costs are high and constantly recurring, unlike in standard employment, where job search is performed infrequently. Moreover, in standard employment, much of the search cost is borne by the employer, while in the microtask market, almost all of the search cost is borne by workers. To address this problem, workers used heuristics that allowed them to pick tasks that were likely to be good with minimal search time, or failing that, satisfice and pick a decent task (or an outside option) after a certain period of searching.

Given the small non-probability sample, no firm conclusions can be drawn about the prevalence of the different heuristics identified in this study, but a preference for tasks that come in large batches was particularly prominent among our informants in addition to their having preferred employers. With these findings in mind, and in order to more thoroughly assess how these individual heuristics, with regard to how these workers "turk", as they themselves put it, eventually aggregate collectively at the level of the market, we now turn to an econometric analysis of the problem of task choice in MTurk. 


\section{An econometric analysis of task choice in MTurk}

\subsection{Dataset}

\subsubsection{Data collection}

We parsed the HIT Group list (see example in Figure 1) on MTurk web site every 3 minutes from November $20^{\text {th }}, 2013$ until February $10^{\text {th }}, 2014$, in order to gather the information on available HIT groups and their current level of completion. This list was parsed in descending order of the number of HITs still available within each HIT group.

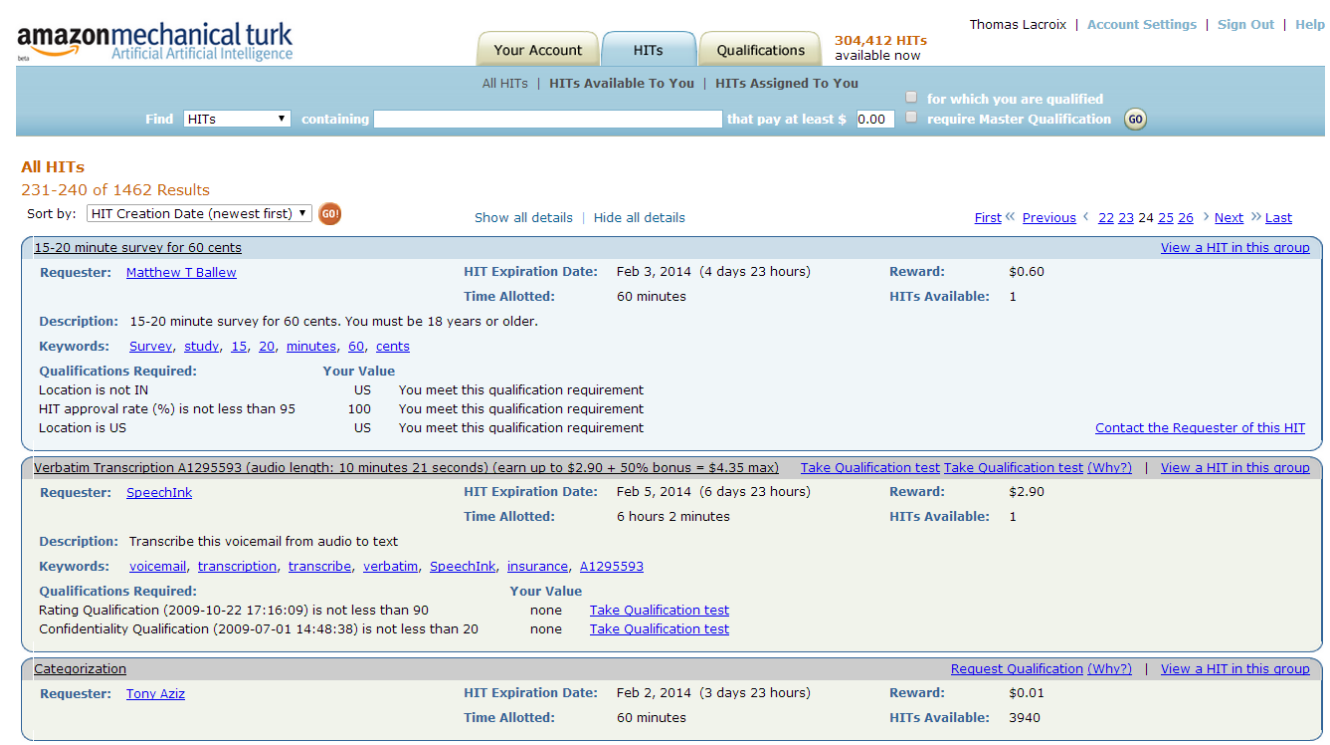

Figure 1 - MTurk HIT list

Each time, we collected the following information for the 200 largest HIT groups.

- $\quad$ HIT id

- Title: short description of HITs within the HIT group

- Requester id

- Requester name

- HIT Expiration Date: the date when HITs will be removed

- Reward: the reward in dollars for successfully completing a single HIT

- Time allotted: how long a Worker can hold on to a HIT

- Number of HITs Available: the number of HITs currently available in the HIT Group

- Description: a more precise description of the HIT

- Keywords: classification of HITs to facilitate search 
- Qualifications Required: the qualifications the worker need to satisfy to be allowed to execute the HITs

We also collected a secondary dataset from Turkopticon (Irani \& Silberman, 2013; Benson et al., 2013). Turkopticon is a community-maintained browser plugin which allows workers to rate requesters along various dimensions. These dimensions, rated from 1 to 5, are:

- Quality of requester communications,

- how well the requester pays,

- how fairly the requester treats workers, and

- how quickly the requester pays for completed HITs, and

- number of reviews.

In order to study a homogenous sample of workers, we limited our sample to HIT groups that had requested the 'Masters have been granted' qualification from workers. This qualification is granted by MTurk to recurrent MTurk workers based on models used to identify workers who "consistently demonstrate a high degree of success in performing a wide range of HITs across a large number of Requesters"3. It is available to all requesters and relatively widely used even if it implies that requesters pay a significantly higher overhead (30\% instead of $10 \%)$ to Amazon. Although its precise specifications have not been made publicly available by Amazon, it is widely agreed among MTurk workers that it corresponds to workers who would have completed thousands of jobs over one or more years and whose approval rate (approval of their work by requesters) would be very high. These HIT groups typically also pay more (Kaplan et al., 2018). In total, we gathered information about 3228 HIT Groups using this procedure, which allows us to avoid biases that would have been come from an excessively heterogeneous population notably with respect to non-recurring workers or to workers whose learning curve with respect to MTurk would still be in its non-stationary stages.

\footnotetext{
${ }^{3}$ https://www.mturk.com/worker/help and http://mechanicalturk.typepad.com/blog/2011/06/get-betterresults-with-less-effort-with-mechanical-turk-masters-.html (accessed 2018-07-09)
} 


\subsection{2 "Best part” algorithm}

We further limited our dataset to HIT Groups for which we had more than 200 data points (i.e. more than 3 hours of activity) and developed a "best part" algorithm in order to get rid of gaps in time series and to account for the fact that some HIT groups are closed and "reloaded" with identical content but more HITs. For each given HIT group, we determine when it had the maximum number of HITs available, which becomes the first point of our cleaned-up subsequence while the last point in the subsequence is defined to be the point before both a) the first gap in the data and b) occurrences when the number of available HITs increased.

Figure 2 presents 3 distinct examples of the sample selection procedure, where the "best part" kept for future analysis is marked by a red rectangle on the left-hand side pictures, of which the red-hand side pictures present zooms.

\subsubsection{Mean throughput estimation}

To measure the attractiveness of HIT groups for workers, we use a measure for the intensity of HIT completion by computing the difference between the maximum number of HITs available and the minimum number of HITs available in each cleaned subsequence using our "Best Part" algorithm and divide it by its duration. This approach is similar to that of Difallah et al. (2015) and Dube et al. (2018) who also measure the throughput of HITs (but do not limit their analysis to the best-part sub-sequences). It differs from the approaches taken in Ipeirotis (2010) and Wang et al. (2011), who utilize survival type models to study completion of HIT groups. We argue that the throughput during the best part is a more natural measure for HIT attractiveness because it is robust in situations where the employer closes a HIT group before its completion, and creates a new identical HIT group ${ }^{4}$, and in situations where the requester removes a HIT group before all HITs within the group are completed.

\footnotetext{
${ }^{4}$ Chilton et al. (2010) suggest that some requesters may "game" the MTurk UI by "reloading" their HIT groups automatically in order to stay on top of the search results.
} 


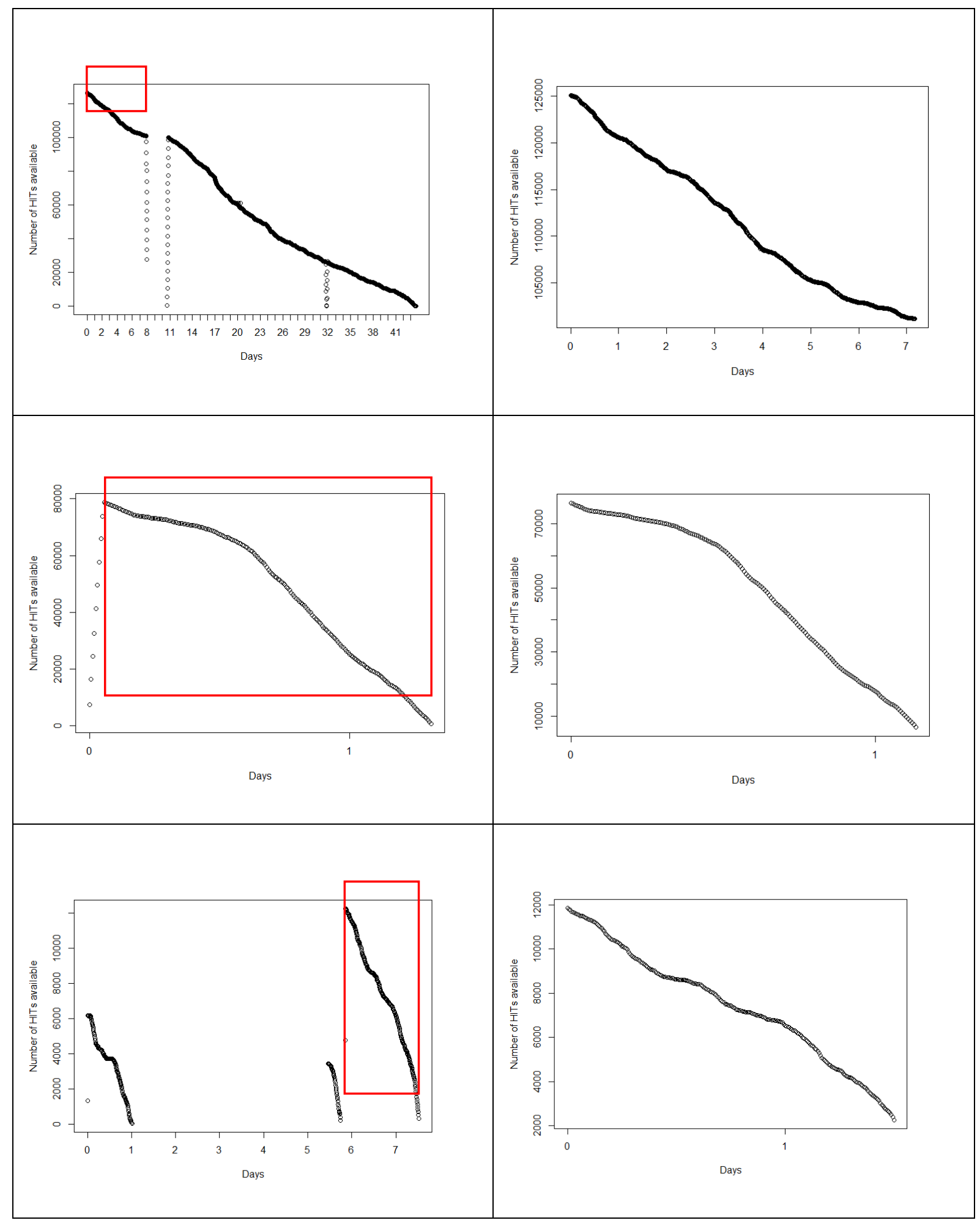

Figure 2 - "Best part" algorithm: 3 examples 


\section{Models}

\subsection{Cross-sectional model}

We now turn to studying the determinants of HIT throughput and begin by estimating variants of the following regression specification:

$\log \left(\right.$ throughput $\left._{\mathrm{i}}\right)=\alpha+\beta_{1} \log \left(\right.$ MaxHitsAvailable $\left._{i}\right)+\beta_{2} \log \left(\right.$ Reward $\left._{i}\right)+$ $\beta_{3} \log \left(\right.$ TitleLength $\left._{i}\right)+\beta_{4} \log \left(\right.$ DescrLength $\left._{i}\right)+\beta_{5}$ CommunicationScore $_{i}+$ $\beta_{6}$ PaymentScore $_{i}+\beta_{7}$ FairnessScore $_{i}+\beta_{8}$ QuicknessScore $_{i}+\beta_{9} \log \left(\right.$ Reviews $\left._{i}\right)+\varepsilon_{i}$.

Columns (1)-(3) of Table 2 present estimation results from a series of specifications without applying control variables from Turkopticon. The association between MaxHitsAvailable and throughput is found to be positive and statistically significant. The interpretation is clear: larger HIT groups attract more workers to work on them. More surprisingly, the association between throughput and price is found to be noisy and indistinguishable from zero at conventional risk levels. Column (4) presents the same model with extra controls for requester reputation downloaded from Turkopticon. The results for the main variables remain largely constant; including covariates derived from Turkopticon does not qualitatively change the parameter estimates despite causing a significant bump in R2. In particular, it is noticeable that being a more experienced requester (measured by number of reviews) is positively associated with throughput. Clearly, a possible interpretation for this is that more experienced requesters on average are better at setting up their HITs in a way that they are quickly filled and have thus acquired a positive reputation over time, which is supported by the findings of Section 3 and the fact that all 10 interviews had "preferred" requesters on whose HITs they preferred to work on.

However, the specification presented in Table 2 could be biased by various types of omitted variables. Firstly, there could be unobservable factors in large HIT groups that would increase throughput. This could happen, for instance if larger HIT groups are systematically easier, and failing to control for that, the association between throughput and maximum HITs available would bias the parameter estimate for $\beta_{1}$ systematically upward. A similar omitted variable bias could be introduced when estimating the association between reward and throughput, denoted $\beta_{2}$. Requesters might know that a HIT group is particularly simple and therefore they would not face difficulties in getting the HITs completed, regardless of the reward. According to this logic, the estimate for $\beta_{2}$ could be biased downwards. We now address these issues using a panel data specification. 
Table 2: Pooled cross sectional specifications

\begin{tabular}{|c|c|c|c|c|}
\hline & \multicolumn{4}{|c|}{ Dependent variable: } \\
\hline & \multicolumn{4}{|c|}{ Throughput (log) } \\
\hline & (1) & (2) & (3) & (4) \\
\hline \multirow[t]{2}{*}{ MaxHitsAvailable (log) } & $0.825^{* * *}$ & & $0.870^{* * *}$ & $0.797^{* * *}$ \\
\hline & $(0.151)$ & & $(0.157)$ & $(0.179)$ \\
\hline \multirow[t]{2}{*}{ Reward (log) } & & -0.096 & 0.197 & 0.040 \\
\hline & & $(0.212)$ & $(0.190)$ & $(0.206)$ \\
\hline \multirow[t]{2}{*}{ TitleLength (log) } & -0.520 & -0.539 & -0.565 & -0.497 \\
\hline & $(0.428)$ & $(0.499)$ & $(0.430)$ & $(0.426)$ \\
\hline \multirow[t]{2}{*}{ DesrcLength (log) } & 0.135 & 0.180 & 0.076 & 0.114 \\
\hline & $(0.332)$ & $(0.389)$ & $(0.336)$ & $(0.334)$ \\
\hline \multirow[t]{2}{*}{ Communication Score } & & & & -0.006 \\
\hline & & & & $(0.099)$ \\
\hline \multirow[t]{2}{*}{ Payment Score } & & & & 0.256 \\
\hline & & & & $(0.162)$ \\
\hline \multirow[t]{2}{*}{ Fairness Score } & & & & -0.111 \\
\hline & & & & $(0.230)$ \\
\hline \multirow[t]{2}{*}{ Quickness Score } & & & & 0.262 \\
\hline & & & & $(0.231)$ \\
\hline \multirow[t]{2}{*}{ Reviews (log) } & & & & $0.340^{*}$ \\
\hline & & & & $(0.183)$ \\
\hline \multirow[t]{2}{*}{ Constant } & $-6.676^{* * *}$ & -0.993 & $-6.002^{* * *}$ & $-8.174^{* * *}$ \\
\hline & $(2.036)$ & $(2.245)$ & $(2.136)$ & $(2.231)$ \\
\hline Observations & 91 & 91 & 91 & 81 \\
\hline $\mathrm{R}^{2}$ & 0.267 & 0.017 & 0.276 & 0.424 \\
\hline
\end{tabular}




\subsection{Panel data specification}

We searched for occurrences of HIT groups that had been posted several times with different maximum HITs available or different prices but with same title, description and requester and qualification for workers. These repeated observations of HIT groups allow us to re-estimate the cross-sectional model using HIT group fixed effects. This specification gets the form:

$$
\log \left(\text { throughput }_{\mathrm{i}}\right)=\alpha_{i}+\beta_{1} \log \left(\text { MaxHitsAvailable }_{i}\right)+\beta_{2} \log \left(\text { Reward }_{i}\right)+\varepsilon_{i}
$$

Panel data specification has a HIT group specific intercept term $\alpha_{i}$, which is the HIT Group fixed effect. Including this term in the estimation precludes us from identifying regression coefficients on time-invariant terms. On the other hand, the HIT group fixed effects subsume the time-invariant variation which might bias the estimates in the previous section. In particular, we hypothesize that the difficulty of HIT group, requester budget and other factors which we cannot control for, but which affect price setting, HIT group size and the throughput are controlled by HIT Group fixed effects.

Columns (1) - (3) of Table 3 present the results of this panel specification. The association between maximum HITs available and throughput is found to be large and is almost unchanged in comparison to estimates reported in Table 2. The deviations between panel and crosssectional specifications are larger with regard to the estimates for the association between reward and throughput. Further, the association is found to be larger in the specification that controls for maximum HITs available. This implies that reward and HIT group size are negatively correlated with one another while being positively correlated with throughput. This is, again, consistent with the notion that workers tend to prefer large HIT groups. In addition, comparison between specifications (2) and (3) suggests that rewards and HIT group sizes are partial substitutes: requesters who are not able to post large HIT groups could partially mitigate this handicap by paying higher rewards. To highlight this, Table 4 replicates the analysis of Table 3 with data split into two subsamples according to MaxHitsAvailable. The association between throughput and price is found to be larger and statistically significant in the belowmean subsample as opposed to the above-mean subsample where it is statistically indistinguishable from zero. 
Drawing together the evidence from both sets of specifications, the most salient finding is that larger HIT groups tend to complete quicker, which is perfectly consistent with the conclusions of Section 3 on the workers' strong preference towards working on batch HITs. The reward for individual HITs has a much less pronounced effect on throughput. The regression coefficient is only significant in the below-mean subsample. This is again consistent with the prevalence of worker heuristics reported above. Taken together, the results suggest that workers prefer batch HITs which they can concentrate on for extended periods of time. At the same time, the reward for individual HIT is found to be a much weaker predictor of HIT group completion.

Table 3: Panel data specifications

\begin{tabular}{|c|c|c|c|}
\hline & \multicolumn{3}{|c|}{ Dependent variable: } \\
\hline & \multicolumn{3}{|c|}{ Throughput (log) } \\
\hline & (1) & $(2)$ & (3) \\
\hline MaxHitsAvailable (log) & $0.949^{* * * *}$ & & $0.965^{* * *}$ \\
\hline & $(0.120)$ & & $(0.115)$ \\
\hline \multirow[t]{2}{*}{ Reward (log) } & & 0.318 & $0.501^{*}$ \\
\hline & & $(0.277)$ & $(0.269)$ \\
\hline Observations & 611 & 611 & 611 \\
\hline $\mathrm{R}^{2}$ & 0.859 & 0.770 & 0.863 \\
\hline Note: & \multicolumn{3}{|c|}{${ }^{*} \mathrm{p}<0.1 ;{ }^{* *} \mathrm{p}<0.05 ;{ }^{* * *} \mathrm{p}<0.01$} \\
\hline
\end{tabular}


Table 4: Panel data specifications (split-sample)

\begin{tabular}{|c|c|c|}
\hline & \multicolumn{2}{|c|}{ Dependent variable: } \\
\hline & \multicolumn{2}{|c|}{ Throughput (log) } \\
\hline & (1) & $(2)$ \\
\hline MaxHitsAvailable (log) & $0.921^{* * *}$ & $0.580^{*}$ \\
\hline & $(0.214)$ & $(0.300)$ \\
\hline \multirow[t]{2}{*}{ Reward (log) } & $0.699^{*}$ & 0.328 \\
\hline & $(0.379)$ & $(0.654)$ \\
\hline & \multicolumn{2}{|c|}{ MaxHitsAvailable MaxHitsAvailable } \\
\hline \multicolumn{3}{|l|}{ Data filter } \\
\hline & $<$ mean & $>=$ mean \\
\hline Observations & 473 & 138 \\
\hline $\mathrm{R}^{2}$ & 0.856 & 0.867 \\
\hline Note: & \multicolumn{2}{|c|}{${ }^{*} \mathrm{p}<0.1 ;{ }^{* *} \mathrm{p}<0.05 ;{ }^{* * *} \mathrm{p}<0.01$} \\
\hline
\end{tabular}

\section{Conclusion}

In the standard model of employment, tasks are bundled into full-time-sized chunks, the exact content of which workers have limited choice over. In recent years, various forms of technologically enabled non-standard working forms have emerged, of which MTurk is an extreme example. On MTurk, workers are in principle free to choose exactly how many tasks of which type and in what sequence to complete. This is expected to allow workers to exercise flexibility over their work, fitting work to individual circumstances. However, even in this 
environment without organizational controls, we find that certain recurring patterns or regularities emerge in workers' task choices. Workers prefer to attach themselves to experienced employers whom they know from before. Workers also clearly prefer long series of repeatable tasks over one-off tasks, even when one-off tasks pay considerably more. Workers also use several other heuristics to guide their task choice. All of these heuristics can be explained as helping the workers to minimize unpaid search time and maximize earnings over time, which is consistent with recent results that have stressed the importance of avoiding unpaid work (Kaplan et al., 2018).

On this what is ostensibly a spot market for tasks, we thus see a re-emergence of certain types of organizational structure. Even if workers could in principle constantly shift working to the highest bidder, it remains more economical in terms of search cost and risk to favor familiar and well-reputed employers. It is also less risky and more conducive to productivity to attach oneself to strings of repeatable tasks rather than jump from task to task. Does this suggest that the standard employment model is, after all, the most efficient institution for organizing work, towards which workers gravitate even in the absence of formal hierarchy? Not exactly. The findings indicate that there is at the same time a preference for the stability and predictability familiar from standard employment, but also for a certain degree of flexibility, specifically in terms of being able to pick up and drop off the work at a moment's notice. Batch tasks are a preferred shape of work, because their repeatable nature offers continuity, while at the same time their short cycle time allows the work to be dropped instantly when other commitments call on the worker.

Due to rapid technological transformation, the organization of work in society is currently in flux. According to one narrative, we are in the process of moving from standard full-time employment towards an economy of disaggregated tasks individually allocated by spot markets to independent worker-entrepreneurs (Malone 2004). However, our findings suggest a third view. Standard employment may be too inflexible, but purely spot market style contracting is probably too unpredictable for workers seeking to make a living for themselves and family members. We thus see that workers and employers gravitate towards an institutional form that offers a combination of flexibility and predictability, with workers preferring batch tasks even at the expense of forgoing higher-paying individual tasks. In slight contrast to literature that sees MTurk and online labor platforms in general as exemplifying the most extreme forms of commodified and marketized work (Standing, 2016; De Stefano, 2016), our findings suggest that there is a degree of de-marketization and re-institutionalization at work even in MTurk. 
Furthermore, the problem of problem choice sheds additional light on recent findings on monopsony, or more generally market power of online labor market platforms studied e.g. by Dube, et al. (2018) and Kingsley, et al. (2015). The "stickiness" of workers to certain types of tasks or certain employers, can give more price setting power to the demand side resulting in monopsony-like pricing strategies by the employers.

Our findings also have implications for the use of MTurk as a platform for empirical research, since they suggest that suggest that certain types of tasks will be completed slower, or certain employers will need to give higher rewards to attract workers. On the other hand, high volume batch HITs might attract a peculiar and non-representative sub-population of professional Turkers leading to concerns about sample representativeness. Indeed, recent research (Coppock, et al 2018) has shown that non-batch HIT experiments tend to generalize well to offline settings, as long as sufficient quality controls are applied in the survey.

Our results on the centrality of batch tasks finally raise a more general issue about whether nonbatch HITs - non-repetitive tasks - could actually be "crowded out" from MTurk, as a consequence of not being selected by most workers. While it is not possible to draw strong conclusions with this study alone, but we argue this would deserve further investigation. At the very least, our results on the specialization to repetitive tasks gives support to the notion that many online micro workers would paradoxically prefer to be back in employers' chains, even if digital and more flexible chains.

\section{Acknowledgements}

The authors are particularly grateful to Mathieu Lacage and Thomas Lacroix, who were respectively instrumental in gathering the dataset, and in developing the scripts and in providing excellent research assistance in the early stages of the research presented in Section 4. Otto Kässi and Vili Lehdonvirta gratefully acknowledge the financial support from the European Research Council (grant number 639652). This paper supersedes the previous version circulated as "A direct empirical investigation of the determinants of online labour supply in Amazon Mechanical Turk".

\section{References}

Ashford, S. J., George, E., \& Blatt, R. (2007). 2 Old Assumptions, New Work. The Academy of Management Annals, 1(1), 65-117. 
Autor, D. H., \& Handel, M. J. (2013). Putting tasks to the test: Human capital, job tasks, and wages. Journal of Labor Economics, 31(S1), S59-S96.

Barley, S. R., \& Kunda, G. (2006). Gurus, hired guns, and warm bodies: itinerant experts in a knowledge economy (2. print., and 1. paperback print). Princeton, NJ: Princeton Univ. Press.

Benson, A., Sojourner, A., \& Umyarov, A. (2015). Can reputation discipline the gig economy? Experimental evidence from an online labor market.

Bergvall-Kåreborn, B., \& Howcroft, D. (2014). Amazon Mechanical Turk and the commodification of labor. New Technology, Work and Employment, 29(3), 213-223.

Carayol, N., \& Dalle J.-M. (2007). Sequential problem choice and the reward system in Open Science. Structural Change and Economic Dynamics, 18 167-191.

Chilton, L. B., Horton, J. J., \& Miller, R. C. (2010). Task search in a human computation market. In Proceedings of the ACM SIGKDD Workshop on Human Computation, 1-9. http://doi.org/10.1145/1837885.1837889

Connelly, G. E., and Gallagher, D. G., 2004. Emerging trends in contingent work research. Journal of Management, 30, 959-983.

Coppock, A, Leeper, T. and Mullinix, K. J. (2018). Generalizability of Heterogeneous Treatment Effect Estimates across Samples. Proceedings of the National Academy of Sciences, 201808083. doi:10.1073/pnas.1808083115.

Dalle, J. M., \& David, P. A. (2005). The allocation of software development resources in 'open source' production mode. In: Feller, J., Fitzgerald, B., Hissam, S., \& Lakhani, K., eds., Making Sense of the Bazaar, MIT Press.

Davis-Blake, A., and Broschak, J. P., 2009. Outsourcing and the Changing Nature of Work. Annual Review of Sociology 35: 321-340.

Den Besten, M., Dalle, J.-M., \& Galia, F. (2008). The allocation of collaborative efforts in open-source software. Information Economics and Policy, 20(4): 316-322. 
De Stefano, V., (2016) The rise of the "just-in-time workforce": On-demand work, crowdwork and labor protection in the "gig-economy". Conditions of Work and Employment Series No. 71. Geneva: ILO.

Diamond, P. (1982). Wage Determination and Efficiency in Search Equilibrium. The Review of Economic Studies, 49(2), 217-227. Retrieved from http://www.jstor.org/stable/2297271

Difallah, D. E., Catasta, M., Demartini, G., Ipeirotis, P. G., \& Cudré-Mauroux, P. (2015). The Dynamics of Micro-Task Crowdsourcing. In Proceedings of the 24th International Conference on World Wide Web - WWW '15 Companion (pp. 617-617). http://doi.org/10.1145/2740908.2744109

Dube A., Jacobs, J., Naidu, S. \& Suri, S. (2018). Monopsony in online labor markets. NBER Working Paper 24216.

Farenzena, D. S., da Cunha Lamb, L., \& Araújo, R. M. (2016). The cost of search and evaluation in online problem-solving social networks with financial and non-financial incentives. First Monday, 21(8).

Finnerty, A., Kucherbaev, P., Tranquillini, S., \& Convertino, G. (2013). Keep It Simple: Reward and Task Design in Crowdsourcing. In Proceedings of the Biannual Conference of the Italian Chapter of SIGCHI, 14:1--14:4. CHItaly '13. New York, NY, USA: ACM. doi:10.1145/2499149.2499168.

Fraser, J., \& Gold, M. (2001). 'Portfolio Workers': Autonomy and Control amongst Freelance Translators. Work, Employment \& Society, 15(4), 679-697.

Gold, M., \& Mustafa, M. (2013). "Work always wins": client colonisation, time management and the anxieties of connected freelancers. New Technology, Work and Employment, 28(3), 197-211.

Hollister, M., 2011. Employment Stability in the U.S. Labor Market: Rhetoric versus Reality. Annual Review of Sociology 37: 305-324. 
Ipeirotis, P. G. (2010). Analyzing the Amazon Mechanical Turk marketplace. XRDS: Crossroads, The ACM Magazine for Students, 17(2), 16. http://doi.org/10.1145/1869086.1869094

Irani, L. C., \& Silberman, M. S. (2013). Turkopticon: Interrupting worker invisibility in amazon mechanical turk. Proceedings of the SIGCHI Conference on ..., 611-620. http://doi.org/10.1145/2470654.2470742

Kalleberg, A. L., 2000. Nonstandard employment relations: Part-time, temporary and contract work. Annual Review of Sociology, 26: 341-365.

Kalleberg AL (2011) Good Jobs, Bad Jobs: The Rise of Polarized and Precarious Employment Systems in the United States, 1970s-2000s. New York: Russell Sage. Acemoglu, Daron, and David Autor. 2011. Skills, Tasks and Technologies: Implications for Employment and Earnings. Handbook of Labor Economics. Vol. 4. doi:10.1016/S0169-7218(11)02410-5.

Kaplan, T., Saito, S., Hara, K., \& Bigham, J. P. (2018). Striving to Earn More: A Survey of Work Strategies and Tool Use Among Crowd Workers. In HCOMP (pp. 70-78).

Kenney, M., \& Zysman, J. (2016). The rise of the platform economy. Issues in Science and Technology, 32(3), 61 .

Kingsley, S. C., Gray, M. L. \& Suri, S. (2015). “Accounting for Market Frictions and Power Asymmetries in Online Labor Markets." Policy and Internet 7 (4): 383-400. doi:10.1002/poi3.111.

Mason, W. and Watts, D.J. (2010) 'Financial incentives and the performance of crowds', $A C M$ SigKDD Explorations Newsletter, Vol. 11, No. 2, pp.100-108.

Merton, R.K., 1957. Priorities in scientific discovery. American Sociological Review 22 (6), 635-659.

Peirce, C.S., 1896. The economy of research, reprinted in: Hartshorne, C., Weiss, P. (Eds.), 1960. Lessons from the History of Science, Collected Papers of Charles Sanders Peirce, vol. I, pp. 48-49. Harvard University Press, Cambridge.

Pissarides, Christopher A. 1984. "Search Intensity, Job Advertising, and Efficiency." Journal of Labor Economics 2 (1): 128. doi:10.1086/298026. 
Standing G (2016) The Corruption of Capitalism: Why rentiers thrive and work does not pay. London: Biteback Publishing

Wang, J., Faridani, S., \& Ipeirotis, P. (2011). Estimating the completion time of crowdsourced tasks using survival analysis models. Crowdsourcing for search and data mining (CSDM 2011), 31 .

Ziman, J., 1987. The problem of problem choice. Minerva 25, 92-106.

Zuckerman, H.A., 1978. Theory choice and problem choice. Sociological Inquiry 48, 65-95. 